\title{
Efeitos do Uso Crônico do Tramadol sobre a Prenhez da Rata Albina
}

\author{
Effects of chronic use of Tramadol on Pregnant Albino Rats
}

\begin{abstract}
Alexandra Silva Santos ${ }^{1}$, Eliel Nina de Azevedo ${ }^{1}$, Ricardo Martins Oliveira-Filho ${ }^{2}$, Manuel de Jesus Simões ${ }^{3}$, Luiz Kulay Júnior ${ }^{4}$
\end{abstract}

\begin{abstract}
RESUMO
Objetivos: analisar os efeitos do cloridrato de tramadol sobre o binômio materno-fetal da rata albina.

Métodos: foram constituidos 5 grupos de 10 ratas prenhes tratadas, desde o $1^{\circ}$ até o $20^{\circ}$ dia de prenhez, da seguinte forma: GI = controles intactas; GII = controles que receberam 0,5 $\mathrm{ml}$ de água destilada (veículo do fármaco), 1 vez ao dia, por gavagem; GIII, GIV e GV = grupos que receberam respectivamente 6,7, 20,1 e 45,6 $\mathrm{mg} / \mathrm{kg}$ por dia de cloridrato de tramadol dissolvidos em água destilada, uma vez ao dia, por gavagem, sempre em volume de $0,5 \mathrm{~mL}$. $O$ ganho de peso durante a gestação foi acompanhado por pesagens no dia 0 e nos dias 7o, 14ํ e 20 da prenhez. Após sacrifício por depressão respiratória com éter anestésico, quantificouse o número de implantações, de reabsorções, de fetos, de placentas, de malformações maiores, de mortalidade materna e fetal e os pesos dos fetos e das placentas.

Resultados: O ganho de peso corporal materno durante a gestação foi significativamente afetado pelo tramadol, e o efeito foi mais evidente nos grupos IV e V (reduções médias de 41 e 56\% no ganho de peso, respectivamente). No grupo III, o ganho foi mais afetado aos 7 e 14 dias (33\% em média) do que aos 20 dias de gestação (19\%). O tratamento com o fármaco afetou significativamente, e de forma dose-dependente, os seguintes parâmetros: peso individual dos fetos ( $G V=-39,2 \%)$, peso da ninhada ( $G I V=-51,7 \%$; $G V=-44,2 \%)$, número de placentas (GIV =-28,4\%; GV =-11,6\%), peso individual das placentas $(G V=-10 \%)$ e peso do conjunto das placentas ( $G I V=-28,4 \% ; G V=-16,8 \%$ ). Apesar de ter havido aumento do número de reabsorções e de natimortos nos grupos tratados, os dados não foram significativamente diferentes dos controles.

Conclusões: O tramadol tem definidos efeitos deletérios sobre a prenhez da rata albina, afetando não só a matriz como também o concepto. Os efeitos foram, de modo geral, mais expressivos aos 14 do que aos 20 dias de prenhez, sugerindo a fase organogênica do concepto como mais susceptivel à ação do tramadol do que as fases inicial (embriogênica) e final (termo). Os resultados chamam ainda a atenção para os cuidados a serem observados quanto ao uso deste opióide na gravidez.
\end{abstract}

PALAVRAS-CHAVE: Drogas na gravidez. Analgésicos. Peso fetal. Modelos experimentais

${ }^{1}$ Departamento de Obstetrícia e Ginecologia, Faculdade Medicina, Universidade Federal do Pará

${ }^{2}$ Departamento de Farmacologia, Instituto de Ciências Biomédicas, Universidade de São Paulo

${ }^{3}$ Departamento de Morfologia, Disciplina de Histologia, Escola Paulista de Medicina, Universidade Federal de São Paulo

${ }^{4}$ Departamento de Obstetrícia, Escola Paulista de Medicina, Universidade Federal de São Paulo

Correspondência:

Luiz Kulay Júnior

Hospital São Paulo, Departamento de Obstetrícia

Rua Napoleão de Barros $715,8^{\circ}$ andar

04024-002 - São Paulo - SP

\section{Introdução}

Desde os primórdios da civilização, uma das grandes preocupações do ser humano é a supressão da dor. Em algumas circunstâncias esta pode ceder por simples sugestão e, em outras, ser de dificil solução. Na realidade, a dor transcende o conceito sintomático, pois determina resposta global ao estresse ${ }^{1}$.

Na gravidez o trauma não é incomum. O rá- 
pido atendimento atenua as reações que também comprometem o concepto ${ }^{2}$. O feto, por sua vez, possui todo o aparato anatômico, neuroquímico e funcional que o torna apto a receber, transmitir e integrar o estímulo nociceptivo, ou seja, também sente dor ${ }^{3,4}$.

Entre os analgésicos mais importantes situa-se o grupo dos opióides, denominados puros ou verdadeiros, que atuam em receptores específicos $(\mu)$ do sistema nervoso central (SNC) modificando a interpretação da dor, independente do processo algógeno ${ }^{5}$. Neste grupo, o tramadol é um analgésico opióide sintético com dois mecanismos de ação sinérgicos: tem efeito agonista opióide parcial e inibe a recaptura de monoaminas neurotransmissoras ${ }^{6,7}$. Sua eficácia analgésica tem sido considerada comparável à da morfina e do alfentanil, e superior à da pentazocina ${ }^{6}$, com baixo potencial de abuso, de tolerância e de dependência ${ }^{6,7}$. Seu uso em obstetrícia, porém, tem-se restringido à analgesia durante o trabalho de parto ${ }^{8-10}$. Os efeitos adversos mais comuns (cerca de 2 a $6 \%$ de incidência) são náusea, tontura, sonolência, sudação, vômitos e boca seca ${ }^{7}$.

Devido à falta de relatos na literatura sobre a ação deste opióide no curso da gravidez, este trabalho estuda os efeitos do cloridrato de tramadol sobre o binômio feto-materno durante a prenhez da rata albina.

\section{Material e Métodos}

Utilizamos ratas (EPM-1 Wistar) adultas, virgens, pesando de 180 a $200 \mathrm{~g}$, com regime de alimentação (ração Purina-Labina) e de água à vontade. Foram inicialmente acasaladas na proporção de duas fêmeas para cada macho por um período de duas horas. O diagnóstico de prenhez foi feito pelo encontro de espermatozóides na luz vaginal. Cinqüenta ratas foram então divididas aleatoriamente em cinco grupos iguais, como segue: Grupo I - animais não manuseados durante toda a prenhez (controle I); Grupo II - animais que receberam diariamente $0,5 \mathrm{ml}$ de água destilada por gavagem (controle II). As ratas dos grupos experimentais receberam dose única diária de $6,7 \mathrm{mg} /$ $\mathrm{kg}$ de peso corporal (Grupo III), 20,1 mg/kg (Grupo IV) ou $45,6 \mathrm{mg} / \mathrm{kg}$ (Grupo V) de cloridrato de tramadol dissolvido em água destilada, por gavagem. O tratamento teve início logo após a positividade do teste de prenhez ('dia 0') e se estendeu até ao 20 dia; o fármaco foi administrado sempre em volume final de 0,5 $\mathrm{ml}$. Durante todo o experimento foi monitorada a incidência de mor- talidade das matrizes.

O peso corporal das matrizes foi determinado no início do experimento ('dia 0') e no 7은 14ㅇe $20^{\circ}$ dia de prenhez, calculando-se então o ganho de peso porcentual ao longo do período de observação.

Ao termo (20 dia) os animais foram anestesiados com éter etílico. A laparotomia foi realizada por incisão abdominal mediana xifopúbica para exposição dos órgãos internos. Em seguida foi feita a histerotomia, para a retirada de fetos e placentas, como também para contagem dos sítios de implantação, de reabsorções, de placentas e de fetos vivos; foi anotada também a mortalidade fetal. Neste momento, as matrizes, ainda sob anestesia, foram sacrificadas por secção do miocárdio. A seguir foi determinado tanto o peso individual quanto o peso conjunto dos fetos vivos e das placentas em balança analítica (Gehaka B6 1000). Os fetos vivos e mortos foram ainda observados sob lupa estereoscópica para verificar a presença de eventuais malformações externas maiores, principalmente hipospadia, espinha bífida, encurtamento dos membros, polidactilia e lábio leporino/fenda palatina, que correspondem às dismorfoses mais freqüentes.

Os dados ponderais foram submetidos a análise de variância; comparações múltiplas foram feitas pelo teste de Kruskal-Wallis. Estabeleceuse o mínimo de $5 \%$ de significância para rejeição da hipótese de nulidade.

\section{Resultados}

Nos Grupos I (ratas intactas) e II (veículo do tramadol), os ganhos de peso corporal materno ao longo da prenhez, calculados em relação ao peso do 'dia 0', foram essencialmente idênticos e estão representados em conjunto, na Figura 1. Observa-se que a administração de tramadol durante toda a prenhez acarretou significativa queda do ganho de peso. Nos Grupos III e IV $(6,7$ e $20,1 \mathrm{mg} / \mathrm{kg}$ de tramadol por dia, respectivamente) as quedas foram mais pronunciadas até o $14^{\circ}$ dia da prenhez, e menos intensas no último terço da gestação (Tabela 1). Já com a dose maior de tramadol (Grupo V) a interferência com o ganho de peso foi grande e constante (cerca de 56\%) ao longo de todo o período experimental.

Nossos resultados mostraram que o tramadol exerceu importantes efeitos tóxicos sobre diversos parâmetros da rata prenhe (Tabela 2). Nos grupos que receberam o fármaco encontramos um total de 3 natimortos contra apenas 1 nos grupos controle; igualmente, verificamos aumento ex- 
pressivo do número de reabsorções por efeito do tramadol. Estes dados, entretanto, não alcançaram significância estatística, provavelmente em função da sua dispersão.

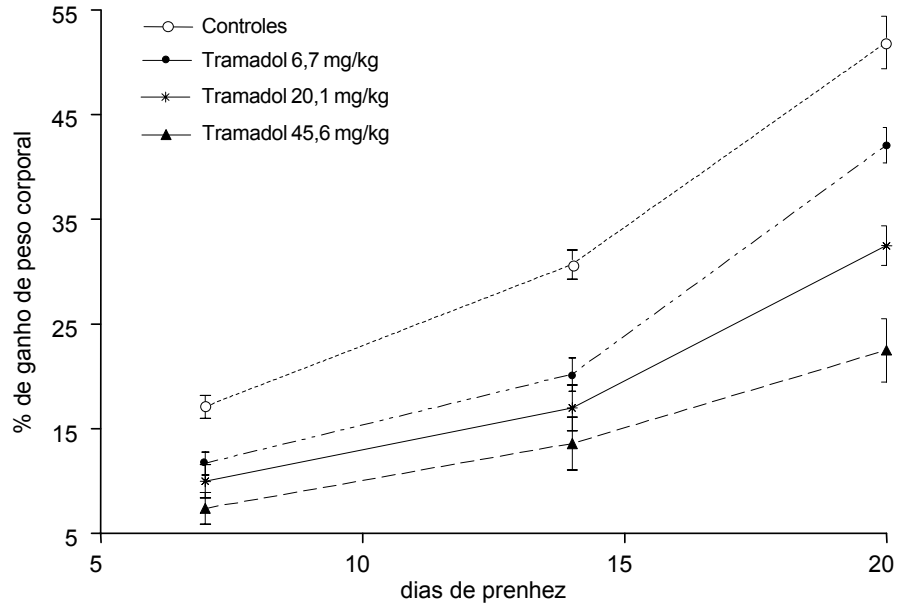

Figura 1 - Ganho de peso corporal de ratas em relação ao 'dia 0' de prenhez. Os valores englobados dentro do mesmo par de colchetes não são estatisticamente diferentes. Para todos os demais valores, comparados no mesmo dia ou entre dias diferentes, $p<0,05$
Tabela 1 - Porcentagem de redução do ganho de peso materno ao longo da prenhez da rata albina causada pela administração de cloridrato de tramadol, calculada em relação ao desenvolvimento dos grupos controle Gl e GII combinados. Nos grupos III, IV e V os animais receberam respectivamente 6,7,20,1 e 46,6 mg/kg de cloridrato de tramadol por gavagem, uma vez ao dia, desde o primeiro até $020^{\circ}$ dia de prenhez. Os valores são de médias ( \pm EPM) para 10 animais em cada grupo.

\begin{tabular}{|c|c|c|c|}
\hline \multirow[t]{2}{*}{$\%$ de red } & \multicolumn{3}{|c|}{$\%$ de redução do incremento de peso corporal } \\
\hline & $7^{\circ} \mathrm{dia}$ & & \\
\hline , ingengy & & & \\
\hline g & 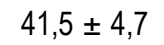 & 9 & 37 \\
\hline madol 45,6 mg/kg & $56,7 \pm 7,6$ * & $55,7 \pm 6,4$ * & $56,6 \pm 5,1$ * \\
\hline
\end{tabular}

A análise estatística revelou as seguintes diferenças significativas: ${ }^{*} p<0,01$ em relação a GIII;

${ }^{* *} p<0,001$ em relação a $G I I I ;$

${ }^{\dagger} p<0,01$ em relação a GIV.

Tabela 2 - Efeitos do tramadol sobre parâmetros da prenhez da rata albina. Os Grupos I e II são grupos controle (I = intacto; II = veículo do fármaco). Nos Grupos III, IV e V os animais receberam respectivamente 6,7,20,1 e 46,6 mg/kg de cloridrato de tramadol por gavagem, uma vez ao dia, desde o primeiro até $020^{\circ}$ dia de prenhez. Os valores são média \pm EPM obtidos em 10 animais para cada grupo.

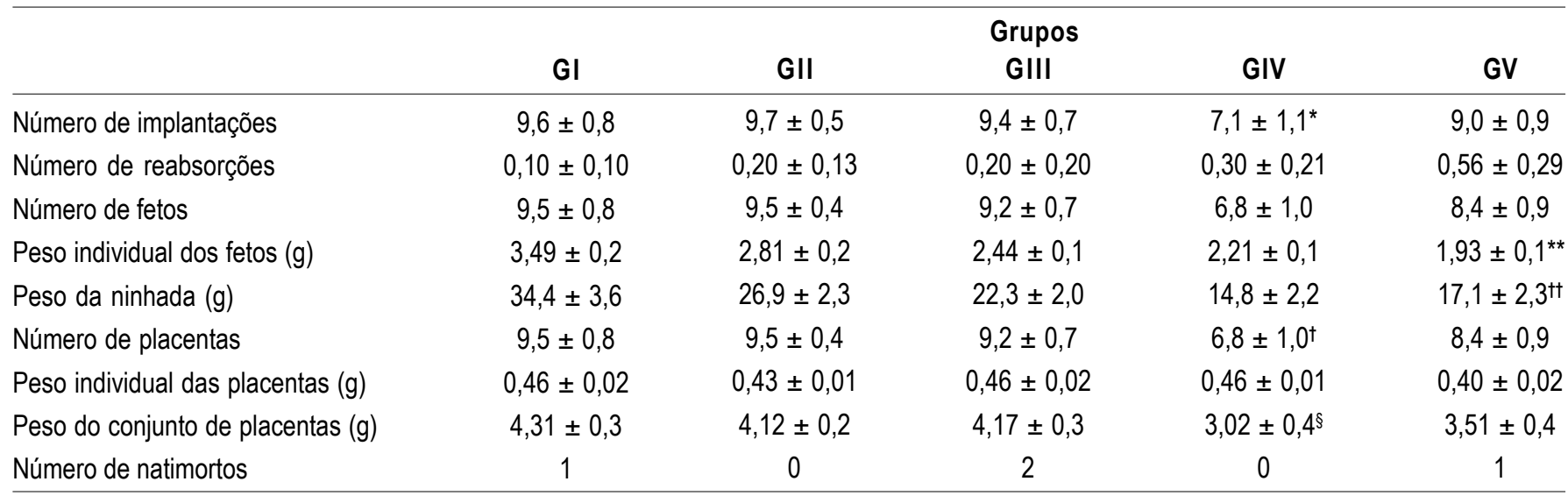

A análise estatística revelou as seguintes diferenças significativas: * $p<0,05$ em relação a GII e GIII; * $p<0,001$ em relação a GI, GII e GIII; ${ }^{\dagger} p<0,05$ em relação a GI e GII; ${ }^{\dagger \dagger} p<0,001$ em relação

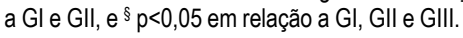

Com relação a todos os demais parâmetros, contudo, os efeitos foram altamente significativos (Tabela 2). Particularmente dignos de nota foram as reduções observadas no peso individual fetal e no peso da ninhada, que apresentaram valores de queda de até $50 \%$ em relação aos grupos controle. Menos intensamente, embora ainda significativamente afetados, foram o número de implantações, o número de fetos e os dados referentes ao compartimento placentário.

\section{Discussão}

A faixa de doses de tramadol usadas no presente trabalho $(6,7$ a $45,6 \mathrm{mg} / \mathrm{kg}$, via oral) situase muito aquém dos valores de $\mathrm{DL}_{50}$ relatados em estudos de toxicidade aguda ${ }^{11}$. Contudo, distúrbios de comportamento foram relatados com doses bem mais baixas $(25 \mathrm{mg} / \mathrm{kg})$, porém administradas por via intravenosa, sem sinais de alterações patológicas, lesões morfológicas ou achados neuropatológicos ${ }^{11}$. A ausência de malformações maiores atribuiveis ao tramadol administrado ao 
longo de toda a prenhez da rata confirma os dados prévios da literatura sobre a aparente ausência de risco genotóxico do fármaco ${ }^{11}$.

É bem conhecido o fato de o ganho de peso corporal durante o evolver da prenhez da rata não ser homogêneo ${ }^{12}$, e isto pode ser visto pelos dados ponderais verificados em nossos grupos controle. O tratamento com três diferentes doses de tramadol durante todo o periodo da prenhez produziu definida e significativa diminuição na velocidade do ganho de peso.

É interessante a observação de que, com as duas doses menores (Grupos III e IV), o tramadol mostrou-se mais deletério sobre o ganho de peso nos primeiros 15 dias, passando a não mostrar efeito tão exuberante no terço final da prenhez, mais perto do termo. É provável que, nessa faixa de doses (6 a $20 \mathrm{mg} / \mathrm{kg}$ ), a toxicidade do tramadol seja limítrofe e afete, de modo dosedependente, a fase organogênica do concepto. $\mathrm{Na}$ fase final, mais próxima do termo, o efeito do fármaco talvez seja devido mais a fatores nutricionais do que propriamente a uma toxicidade específica. De fato, o propoxifeno, também analgésico opióide, tem como efeito colateral a redução do peso corporal a partir da segunda semana de tratamento ${ }^{13,14}$. Por outro lado, no grupo de ratas tratadas com a dose mais elevada $(45,6 \mathrm{mg} /$ $\mathrm{kg})$, houve grave restrição ao ganho de peso em todos os períodos da prenhez, sendo neste caso perdida a relação dose-efeito.

Os opiáceos, entre suas múltiplas ações, retardam a passagem do bolo alimentar do estômago para o duodeno em até 12 horas, além de provocarem diminuição das secreções biliares, pancreáticas e intestinais, acarretando sensação de saciedade ${ }^{15,16}$. Além disso, em nível central, a sedação pode promover vários graus de embotamento da consciência, também com prejuízo da ingestão. De qualquer modo, o efeito tóxico em nivel central talvez seja devido mais à atividade bloqueadora do mecanismo de recaptura monoaminérgica (noradrenalina, serotonina) do que aos efeitos opióide-símiles diretamente relacionados com receptores opióides no $\mathrm{SNC}^{17}$.

Independentemente dos efeitos nutricionais, o tramadol pode causar toxicidade por efeito hepático. Em um sistema de ensaio com hepatócitos de embrião de galinha in vitro, comprovou-se que o tramadol tem potente ação porfirogênica em comparação com analgésicos como nalbufina, oxicodona, buprenorfina ou morfina ${ }^{18}$. Assim, não é improvável que parte do efeito tóxico do tramadol observado neste trabalho seja devido a este fator.

A via metabólica principal para a eliminação do tramadol depende da atividade CYP2D6 do citocromo P-450, a qual dá origem ao seu metabólito ativo O-desmetilado. Há relato na literatura de um caso de grave reação debilitante devida a uma única dose oral de tramadol, em que a paciente, mulher de 32 anos sem história médica digna de nota, revelou-se portadora de atividade CYP2D6 extremamente alta ${ }^{19}$. Assim, é plausível que o metabólito O-desmetiltramadol tenha sido responsável, pelo menos em parte, pelo efeito debilitante do fármaco nos nossos grupos experimentais.

Nos grupos controle, o aporte protéicocalórico para o binômio materno-fetal fluiu normalmente e foi suficiente para atender os requerimentos básicos necessários ao desenvolvimento fetal. Nos grupos experimentais, o retardo do trânsito gastroduodenal do bolo alimentar ${ }^{17}$ nas matrizes, ocasionado provavelmente não só pelo tramadol como também pelo seu metabólito ativo, presumivelmente determinou redução do fluxo de nutrientes essenciais para o desenvolvimento dos conceptos $^{20-22}$. A par disto, é preciso lembrar que os fetos foram expostos ao fármaco durante toda a prenhez, ficando comprometidas, então, tanto a fase de crescimento celular (hiperplasia/ hipertrofia) quanto a do desenvolvimento dos órgãos (hipertrofia) ${ }^{23}$, a exemplo do que ocorre nos estados de desnutrição ${ }^{24,25}$.

Assim, podemos dizer que o tramadol, analgésico opióide, diminuindo a ingestão e retardando o trânsito do bolo alimentar nas ratas, pode ser responsabilizado pelo baixo ganho de peso tanto das matrizes quanto dos conceptos ao nascer, não estando descartada uma possivel participação de seu metabólito ativo O-desmetiltramadol nesse efeito tóxico.

\section{SUMMARY}

Purpose: to examine the effects of tramadol hydrochloride on rat pregnancy.

Methods: five groups of 10 pregnant albino rats each were treated from the 1st up to the 20th day of pregnancy as follows: $G I=$ intact controls; $G I I=$ controls which received $0.5 \mathrm{~mL}$ of distilled water (drug vehicle) once a day by gavage; GIII, GIV and $G V=$ groups treated respectively with 6.7, 20.1 or $45.6 \mathrm{mg} / \mathrm{kg}$ of tramadol hydrochloride once a day by gavage in a final volume of $0.5 \mathrm{~mL}$. Body weight gain was monitored by weighing at the beginning and on the 7th, 14th and 20th day of pregnancy. At term the animals were killed under deep ether anesthesia and the following parameters were evaluated: number of implantations, of resorptions, of viable fetuses and of placentae; presence of major malformations; 
maternal and fetal mortality and weights of fetuses and placentae.

Results: tramadol significantly affected maternal body weight gain, this effect being more apparent in groups IV and $V$ (mean reductions of weight gain of 41 and $56 \%$, respectively). In group III the weight gain was affected more at days 7 and 14 (33\% mean gain reductions) than at day 20 (19\%). Drug treatment affected significantly and in a dose-dependent fashion the following parameters: individual weight offetuses $(G V=-39.2 \%)$, offspring weight $(G I V=-51.7 \% ; G V=-44.2 \%)$, number of placentae $(G I V=$ $-28.4 \% ; G V=-11.6 \%)$, individual weight of placentae $(G V$ $=-10 \%)$ and the total weight of placentae (GIV $=-28.4 \%$; $G V=-16.8 \%$ ). Though among the treated animals there was an increase in resorptions and deaths at birth, these events were not significantly different from those found in controls.

Conclusions: Tramadol showed definite deleterious effects on albino rat pregnancy, and these effects were exerted not only on the maternal but also the on fetal organisms. Overall, the effects were more pronounced at the 14th than at the 20th day of pregnancy, thus suggesting that the organogenic phase of the fetus is more susceptible than its initial (embryogenic) or final (term) phases. The results call attention to the care which is to be taken when the use of this opioid is considered during pregnancy.

KEY WORDS: Tramadol. Drug effects. Opioid analgesics. Fetal weight

\section{Referências}

1. Anand KJ, Carr DB. The neuroanatomy, neurophysiology and neurochemistry of pain, stress and analgesia in newborns and children. Pediatr Clin North Am 1989; 36:795-822.

2. Harris AP, Barton CR, Baker DR. The pregnancy trauma patient. In: Stene JK, Grande CM, editors. Trauma Anesthesia. 1st ed. Baltimore: Williams \& Wilkins; 1991. p.301-11.

3. Charnay Y, Paulin C, Chayvialle JA, Dubois PM. Distribution of substance P-like immunoreactivity in the spinal cord and dorsal root ganglia of the human foetus and infant. Neuroscience 1983; 10:41-55.

4. Nomura H, Shiosaka S, Inagaki S, et al. Distribution of substance P-like immunoreactivity in the lower brainstem of the human fetus: an immunohistochemical study. Brain Res 1982; 252:315-25.

5. Shannon M, Berde CB. Pharmacologic management of pain in children and adolescents. Pediatr Clin North Am 1989; 36:855-71.

6. Kayser V, Besson JM, Guilbaud G. Effects of the analgesic agent tramadol in normal and arthritic rats: comparison with the effects of different opioids, including tolerance and cross-tolerance to morphine. Eur J Pharmacol 1991; 195:37-45.
7. Scott LJ, Perry CM. Tramadol: a review of its use in perioperative pain. Drugs 2000; 60:139-76.

8. Bitsch VM, Emmrich J, Hary J, Lippach G, Rindt W. Geburtshilfliche Analgesie mit Tramadol. Fortschr Med 1980; 98:632-4.

9. Sunshine A, Olson NZ, Zighelboim I, De Castro A, Minn FL. Analgesic oral efficacy of tramadol hydrochloride in pain following cesarean section. Clin Pharmacol Ther 1991; 49:181-3.

10.Urbanetz AA, Marques LO, Brandão SR, Daros VI. Influência do cloridrato de tramadol e da associação meperidina-prometazina no trabalho de parto. Rev Bras Ginecol Obstet 1997; 19:523-9.

11. Matthiesen $\mathrm{T}$, Wohrmann $\mathrm{T}$, Coogan TP, Uragg $\mathrm{H}$. The experimental toxicology of tramadol: an overview. Toxicol Lett 1998; 95:63-71.

12.Baba T, Nagahama M, Akiyama N, Miki T. Experimental production of malformation due to acetyl salicylate and phenyl salicylate in rats. Osaka City Med J 1966; 12:23-29.

13.Emmerson JL, Owen NV, Koenig GR, Markham JK, Anderson RC. Reproduction and teratology studies on propoxyphene napsylate. Toxicol Appl Pharmacol 1971;19:471-9.

14. Mendes ETR, Simões MJ, Evêncio-Neto J, Espiridião S, Kulay Júnior L. Ação crônica do napsilato de propoxifeno na prenhez da rata. Rev Bras Ginecol Obstet 1998; 20: 67-70.

15.Dooley CP, Saad C, Valenzuela JE. Studies of the role of opioids in control of human pancreatic secretion. Dig Dis Sci 1988; 33:598-604.

16.Kromer W. Endogenous and exogenous opioids in the control of gastrointestinal motility and secretion. Pharmacol Rev 1988; 40:121-62.

17.Freye E, Latasch L. Effects of tramadol and tilidine/ naloxone on oral-caecal transit and pupillary light reflex. Arzneimittelforschung 2000; 50:24-30.

18.Lambrecht RW, Gildemeister OS, Williams A, Pepe JA, Tortorelli KD, Bonkovsky HL. Effects of selected antihypertensives and analgesics on hepatic porphyrin accumulation: implications for clinical porphyria. Biochem Pharmacol 1999; 58:887-96.

19.Gleason PP, Frye RF, O'Toole T. Debilitating reaction following the initial dose of tramadol. Ann Pharmacother 1997; 31:1150-2.

20.Naeye RL. Weight gain and outcome of pregnancy. Am J Obstet Gynecol 1979; 135:3-9.

21.Crawford MA. Is there a common denominator between fetal growth retardation and cardiovascular disease? Acta Cardiol 1989; 44:449-53.

22.Dobbing $\mathrm{J}$. The later development of the brain and its vulnerability. In: Davis JA, Dobbing J, editors. Scientific Foundations of Pediatrics. $1^{\text {st }}$ ed. London: Heinemann Medical; 1974. p.565.

23.Winick M. Cellular growth in intrauterine malnutrition. Pediatr Clin North Am 1970; 17:69-78.

24.Burke BS, Beal VA, Kirkwood SB, Stuart HC. Nutrition studies during pregnancy. Am J Obstet Gynecol 1943; 46:38-52.

25.Tonete SSQ, Coelho CAR, Nóbrega FJ. Desnutrição fetal experimental em ratos: efeitos sobre o peso corporal, peso cerebral, o teor de lipídeos totais, proteínas e colesterol no cérebro. J Pediatr 1978; 44:213-21. 